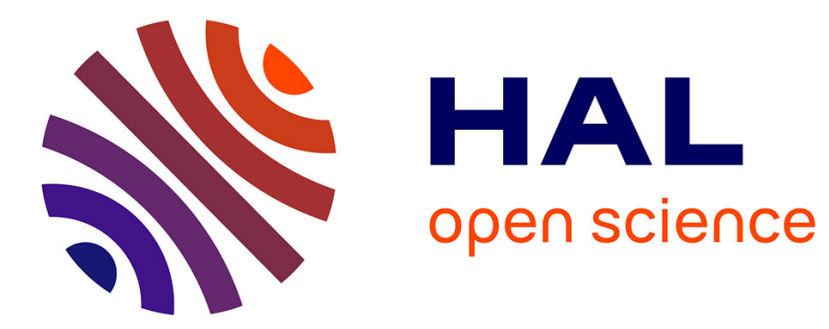

\title{
Scaling approach to spin-glass phenomena
}

S E Barnes, A P Malozemoff, B. Barbara

\section{To cite this version:}

S E Barnes, A P Malozemoff, B. Barbara. Scaling approach to spin-glass phenomena. Physical Review B: Condensed Matter (1978-1997), 1984, 30 (5), pp.2765. hal-01660449

\section{HAL Id: hal-01660449 \\ https://hal.science/hal-01660449}

Submitted on 10 Dec 2017

HAL is a multi-disciplinary open access archive for the deposit and dissemination of scientific research documents, whether they are published or not. The documents may come from teaching and research institutions in France or abroad, or from public or private research centers.
L'archive ouverte pluridisciplinaire HAL, est destinée au dépôt et à la diffusion de documents scientifiques de niveau recherche, publiés ou non, émanant des établissements d'enseignement et de recherche français ou étrangers, des laboratoires publics ou privés. 


\title{
Scaling approach to spin-glass phenomena
}

\author{
S. E. Barnes \\ Physics Department, University of Miami, Coral Gables, Florida 33124
}

A. P. Malozemoff

IBM Thomas J. Watson Research Center, P.O. Box 218, Yorktown Heights, New York 10598

\author{
B. Barbara \\ Laboratoire Louis Néel, Centre National de la Recherche Scientifique, Boîte Postale 166X, \\ F-38042 Grenoble (Cédex), France
}

(Received 30 April 1984)

\begin{abstract}
The scaling model of spin-glass phenomena is extended to consider both longitudinal and transverse order parameters $q_{l}$ and $q_{t}$. The lowest-order coupling in the free energy goes as $q_{l}^{2} q_{t}^{2}$. The field $(h)$ dependence of the reduced temperature $t$ for transverse freezing is found to be $t \sim h^{4 / \delta}$ where $\delta$ is the conventional nonlinear susceptibility field exponent $\left(\chi \sim h^{2 / \delta}\right)$. While the transverse correlation length diverges at $t=0$, the longitudinal correlation length remains finite.
\end{abstract}

\section{INTRODUCTION}

As the present authors have pointed out in previous work, ${ }^{1-4}$ a non-mean-field approach to spin glasses seems to explain many different aspects of spin-glass behavior. For example, it gives a satisfactory explanation of the onset of irreversibility in that it relates this low-temperature ( $T<T_{G}$, the glass temperature) crossover line to the similar high-temperature $\left(T>T_{G}\right)$ line corresponding to the change from analytic to nonanalytic magnetic field dependences. $^{3}$ Experimentally $|t| \sim h^{2 / \phi}$ for both these lines with almost identical values of the exponent $\phi$ and constants of proportionality which differ only by a factor of 2. ${ }^{3}$ [Here $t=\left(T_{G}-T\right) / T_{G}$ and $h=g_{s} \mu_{B} H / k T_{G}$, where $H$ is the magnetic field.]

Here we wish to present a coherent development of this non-mean-field or scaling approach to spin glasses. The development is inspired by that of Suzuki. ${ }^{5}$ The principal difference is the introduction of a third order parameter, an extension of his work to realistic three-component spin systems. The result is a consistent non-mean-field treatment of the transverse freezing, the two crossover lines mentioned above plus the field dependence of the maximum in the magnetization. The mean-field treatment gives a transverse freezing line ${ }^{6} t \sim-h^{2}$ while our approach gives $t \sim-h^{4 / \delta}$. (The experimental value of the high-field susceptibility exponent, $\chi \sim h^{2 / 8}$, is, e.g., $\delta \sim 4-5$ for Cu-Mn.) The above-mentioned results for the crossover lines are derived and, in addition, it is shown that when only the singular part of the magnetization is considered, the maximum has the same exponent as the crossover lines, i.e., $t \sim-h^{2 / \phi}$. However, as the present authors discuss at length elsewhere, ${ }^{4}$ this is modified when the regular part of the magnetization is included; experimental results show $t$ does not even have a monotonic dependence on $h .^{2}$ As is usual with scaling theories, we obtain a multitude of scaling laws. These are standard laws recast in the spin-glass mold. In addition, we obtain predictions for the high-field dependence of the transverse and longitudinal correlation lengths and the magnitude $\bar{m}$ of spin fluctuations.

\section{LANDAU FREE ENERGY}

First, we construct a Landau-type free energy, similar to that of Suzuki, but with three order parameters $m, q_{l}$, and $q_{t}$, the same number as in the microscopic theories: ${ }^{6,7}$

$$
\begin{aligned}
F= & F_{0}+a m^{2}+b m^{4}-c q_{l}^{2}-d q_{l}^{3}-2 c q_{t}^{2} \\
& -2 d q_{t}^{3}+2 f q_{l}^{2} q_{t}^{2}+e q_{l} m^{2}-h m .
\end{aligned}
$$

Here $m=\left\langle S_{i}\right\rangle$ is along the applied field $h$, i.e., $m$ is the usual ferromagnetic order parameter, and $q_{l}$ and $q_{t}$ characterize the longitudinal and transverse spin-glass ordering. The existence of the two glass parameters $q_{l}$ and $q_{t}$ introduces the possibility of a coupling between them. The term $f q_{l}^{2} q_{t}^{2}$ is ultimately justified by reproducing the known mean-field $h^{2}$ shift for the transverse ordering temperature, as will be shown below. The $m^{2} q_{l}$ term was introduced by Suzuki. ${ }^{5}$ A similar $m^{2} q_{t}$ term is not included because the transverse magnetization $m_{t}$ is zero in the absence of a transverse field.

We consider the case of the spin-glass transition temperature well above the ferromagnetic temperature. Thus the coefficient $a$ in Eq. (1) is essentially constant in the range of interest, while

$$
c=c_{0}\left(T-T_{G}\right) \text {. }
$$

The coefficients $a, b, c_{0}, d$, and $e$, along with $q_{l}$ and $q_{t}$, should be taken as positive. Thus the $c q^{2}$ terms are actually negative above the transition temperature and positive below. In an $m-q$ plane the stable free-energy solution must be taken to be a saddle point, whereas in a $q_{l}-q_{t}$ plane the stable free energy must be taken as the maximum. These bizarre conventions and signs arise, according to Suzuki, ${ }^{5}$ from the fact that this free energy is actu- 
ally a zeroth-order replica.

The relationship of the order parameters to the susceptibility is obtained by minimizing $F$ with respect to $m$ :

$$
\frac{\partial F}{\partial m}=0=2 a m+4 b m^{3}+2 e q_{l} m-h
$$

to give

$$
\begin{aligned}
\chi & =\frac{1}{2}\left(a+e q_{l}\right)^{-1} \\
& \simeq(1 / 2 a)\left[1-(e / a) q_{l}\right] .
\end{aligned}
$$

Since experimentally the $T>T_{G}$ inverse susceptibility has a finite intercept $\theta$, we write $2 a=T-\theta$. And if we take $e=a$, we reproduce the Fischer formula ${ }^{8}$

$$
\chi=[1 /(T-\theta)]\left(1-q_{l}\right) .
$$

In a similar way one obtains for $h>\left|4 a c_{0} T_{G}^{0}(3 d e)^{-1 / 2} t\right|$ and in the limit of small $h$ and $t$

$$
q_{l}=(e / 12 a d)^{1 / 2} h
$$

and

$$
q_{t}=\left\{\begin{array}{l}
\left(2 c_{0} / 3 d\right)\left[T_{G}(h)-T\right], q_{t}>0 \\
0, \text { otherwise }
\end{array}\right.
$$

where

$$
T_{G}(h)=T_{G}^{0}-\left(e f / 12 a^{2} c_{0} d\right) h^{2},
$$

which reproduces the $h^{2}$ shift for transverse freezing. ${ }^{6}$ In the limit $h<\left|4 a c_{0} T_{G}^{0}(3 d e)^{-1 / 2} t\right|$,

$$
q_{l}=\left\{\begin{array}{l}
-\left(2 c_{0} T_{G}^{0} / 3 d\right) t-\left(e / 8 a^{2} c_{0}\right)\left(h^{2} / t\right), \quad t<0 \\
\left(e / 8 a^{2} c_{0}\right) h^{2} / t, \quad t>0 .
\end{array}\right.
$$

If we substitute this into the Fischer formula [Eq. (4)] and differentiate to find the maximum [with $d\left(T_{G}^{0}-\theta\right)$ $=2 c_{0} T_{G}^{0}$ as required by mean-field theory], we obtain

$$
t \sim-h^{2 / 3} \text {, }
$$

where we have had to include a $q^{4}$ term in (1) for consistency. This mean-field construction thereby reproduces the famous de Almeida-Thouless ${ }^{9} \frac{2}{3}$ power for the maximum ${ }^{10}$ which in turn is believed to give the crossover to strong replica symmetry breaking. ${ }^{7,11}$

\section{SCALING FREE ENERGY}

To extend the model, we make more or less standard scaling hypotheses for the singular part of $F$ :

$$
\begin{aligned}
F_{s}= & \left(a+e q_{l}\right) m^{2}+b m^{4}-h m-c q_{l}^{2} g^{ \pm}\left(q_{l}|t|^{-\beta}\right) \\
& -2 c q_{t}^{2} g^{ \pm}\left(q_{t}|t|^{-\beta}\right)+f\left(q_{l}, q_{t}\right) .
\end{aligned}
$$

Here $g^{ \pm}(x)$ denotes scaling functions for $t>0$ and $t<0$, respectively, which are assumed to be analytic in $x=q t^{-\beta}$ and to proceed as some power $(\gamma / \beta)$ of $x$ as $x \rightarrow \infty$. Furthermore, $f\left(q_{l}, q_{t}\right)$ is the coupling term whose form we shall determine below.

As before we can assume $a$ and $b$ are constant in temperature, and any temperature dependence of $e$ can be absorbed into $q_{l}$. We arbitrarily identify the temperature dependence of $c$ as $c=c_{0} t^{\gamma}$, which reduces to the meanfield result for $\gamma=1$. Expanding the scaling functions and comparing to the Landau form (1), we can identify $d \sim t^{\gamma-\beta}$; in mean-field with $\gamma=\beta=1$, this temperature dependence vanishes.

Turning now to $f$, we determine its form from the requirement of symmetry with respect to $q_{l}$ and $q_{t}$ and from the assumption, suggested by the mean-field solutions, ${ }^{6,7}$ that a finite field, while suppressing the longitudinal transition, simply shifts the transverse one. The latter implies that the last two terms of Eq. (10) can be combined in the form

$$
-2 c_{0}\left|t^{\prime}\right|^{\gamma} q_{t}^{2} g^{ \pm}\left(q_{t}\left|t^{\prime}\right|^{-\beta}\right),
$$

where now

$$
\begin{aligned}
& t^{\prime}=t-\left[\Delta T_{G}\left(q_{l}\right) / T_{G}^{0}\right], \\
& \Delta T_{G}\left(q_{l}\right)=T_{G}\left(q_{l}\right)-T_{G}^{0},
\end{aligned}
$$

and $T_{G}\left(q_{l}\right)$ is the shifted transition temperature, a function of $q_{l}$ only. Since there is no transverse field, $g^{ \pm}(x)$ in the expression (11) can always be expanded around $x=0$. But in this limit, (11) should reduce to the form of Eq. (10). Therefore, one immediately finds, to lowest order in $q_{l}$

$f\left(q_{l}, q_{t}\right)=-2 c_{0}\left\{\left|t-\left[\Delta T_{G}\left(q_{l}\right) / T_{G}^{0}\right]\right|^{\gamma}-|t|^{\gamma}\right\} q_{t}^{2}$.

This form is not symmetric in $q_{l}$ and $q_{t}$ because, in general, $q_{l} t^{-\beta}$ and $q_{t} t^{-\beta}$ can lie in different limits of their scaling functions $g^{ \pm}\left(q t^{-\beta}\right)$. But when $t \gg \Delta T_{G}\left(q_{l}\right) / T_{G}^{0}$, both $q_{l}$ and $q_{t}$ are near to zero, so Eq. (13) can be expanded to give $t^{\gamma-1} \Delta T_{G}\left(q_{l}\right) q_{t}^{2}$. Then symmetry requires

$$
\Delta T_{G} \propto q_{l}^{2} \text {. }
$$

Furthermore, comparing to the Landau form, we find $f \sim t^{\gamma-1}$, so that in the mean-field limit the temperature dependence vanishes as expected. On the other hand, near the transition line where $t \rightarrow \Delta T_{g}\left(q_{l}\right) / T_{G}^{0}$, Eq. (13) is independent of $q_{l}$ to lowest order and so the transverse freezing has a negligible effect on longitudinal behavior.

\section{GENERALIZED GABAY-TOULOUSE LINE}

Having identified the form of $f\left(q_{l}, q_{t}\right)$, we proceed to solve Eq. (10). As in the earlier mean-field treatment, Eqs. (2)-(4) are unchanged, while the result of differentiating for $q_{l}$ can be written as follows:

$$
\left(e / 4 a^{2}\right) h^{2}-c_{0}|t|^{\gamma+\beta} g_{1}\left(q_{l}|t|^{-\beta}\right)=0 .
$$

Henceforth $g_{i}(x)$ will represent various scaling functions analytic for $x \rightarrow 0$ and proceeding as $x$ to some power for $x \rightarrow \infty$. Equation (15) is inverted to give ${ }^{5}$

$$
q_{l}=|t|^{\beta} g_{2}\left(h^{2}|t|^{-\phi}\right), \quad \phi=\beta+\gamma,
$$

which in turn gives [from Eq. (4)]

$$
\chi=[1 /(T-\theta)]-|t|^{\beta} g_{3}\left(h^{2}|t|^{-\phi}\right) .
$$

In the high-field limit we expect $\chi$ to be temperature independent and to proceed as some power of $h$ which we define by $\chi \sim h^{2 / \delta}$. Then $g_{3}(x) \rightarrow x^{1 / 8}$ as $x \rightarrow \infty$ and the 
scaling law for the crossover exponent is $\phi=\beta \delta$. These results are well known. However, from Eq. (4) the exponent for $q_{l}$ must be the same as that for $\chi$, i.e., $q_{l} \sim h^{2 / \delta}$, and by substituting this in Eq. (14) we find the new and interesting scaling result for the transverse ordering temperature

$$
\left|\Delta T_{G}\right| \propto h^{4 / 8} \text {. }
$$

Since the high-field limit applies when $h \gg|t|^{\phi / 2}$, this result is valid as long as $\phi / 2>\delta / 4$ or $\beta>\frac{1}{2}$. This criterion appears to be fulfilled in all spin glasses studied so far. It would be interesting to compare this prediction to experiment. We are aware of only two very recent studies $^{12}$ on CuMn. Specific-heat measurements of Fogle et al. indicate a transition which first shifted up in temperature to a maximum at a field of about $1 \mathrm{~T}$ (the power law up to this point was not determined), and which later shifted down with an $h^{2}$ dependence. The authors ${ }^{12}$ interpreted these results as evidence for the Gabay-Toulouse mean-field prediction. ${ }^{6}$ In our interpretation, using earlier data on similar CuMn samples, ${ }^{2} \delta \sim 5$; so the temperature shift should proceed as $h^{0.8}$, not far, interestingly, from the crossover power dependence. ${ }^{3}$ Since the critical region is not likely to extend beyond $1 \mathrm{~T}$ in field, ${ }^{1}$ our prediction should be compared to data below $1 \mathrm{~T}$, which is not yet available. Striking, however, is the increase in temperature with field below $1 \mathrm{~T}$; our theory does not exclude such a possibility. On the other hand, the torque measurements of Campbell et al. were performed in more modest fields $(0.5$ to $9 \mathrm{kG})$. They observe no shift in the onset of torque as a function of field which they claim is compatible with the mean-field $h^{2}$ prediction. However, their transition temperature $T^{*}$ is lower, by $10 \%$, than the susceptibility cusp temperature, and so the experimental situation is still far from being explained.

\section{CROSSOVER BEHAVIOR}

Before going on to consider the crossover behavior of the model, it is useful to rewrite the scaling form for the susceptibility:

$$
\chi=g_{0}|t|^{\beta}-h^{2}|t|^{-\gamma} g_{1}\left(h^{2}|t|^{-\phi}\right) .
$$

As has been pointed out earlier, ${ }^{5}$ since $\beta$ and $\gamma$ are positive, it is the coefficient of the nonlinear susceptibility proportional to $h^{2}$ which exhibits a critical singularity. We therefore define

$$
\chi_{\text {sing }}=-h^{2}|t|^{-\gamma} g_{1}\left(h^{2}|t|^{-\phi}\right), \quad \gamma=\phi-\beta
$$

which, with $\phi=\delta \beta$ derived earlier gives Widom's scaling law $\gamma=\beta(\delta-1)$ for this problem. We then use a wellknown scaling theory method. ${ }^{13}$ We define $\bar{\chi}=\left(\chi_{\text {sing }} / h^{2 / \delta}\right)$ and $\bar{t}=\left(t / h^{2 / \phi}\right)$ and note $\bar{\chi} \equiv \chi(\bar{t})$ should be an analytic function of $\bar{t}$, including about $\bar{t}=0$. This provides a connection between $g^{ \pm}(x)$ and makes it unnecessary to distinguish between $t>0$ and $t<0$. About the simplest function one could imagine is

$$
\bar{\chi}(\bar{t})=g_{1} /\left[\bar{t}^{2}+\left(g_{1} / g_{2}\right)^{2 / \gamma}\right]^{\gamma / 2} .
$$

This gives $\bar{\chi} \sim g_{2}$ for $h$ large and $t^{-\gamma}$ for $h$ small, the re- quired asymptotic behavior for $t>0$.

Although our conclusions about crossover behavior will have a more general validity, it is useful to focus attention upon a particular scaling form for $\bar{\chi}$ such as that given above. Clearly a reasonable dividing line between the large and small field limits is when $|\bar{t}|=\left(g_{1} / g_{2}\right)^{1 / \gamma}$, or $t= \pm\left(g_{1} / g_{2}\right)^{1 / \gamma} h^{2 / \phi}$, i.e., there is a "crossover line" dividing the region where $\chi$ is analytic in $h^{2}$ from the one where it is not. The exponent $\phi$ is identified as the crossover (or shift) exponent.

This form of $\bar{\chi}(\bar{t})$ is symmetric but need not be so. One can even imagine $\bar{\chi}(\bar{t})$ becoming multivalued as a way to represent irreversibility. One might also dream up functions with bumps, shoulders, plateaus, glitches, etc. But whatever complications one introduces the line representing the onset of irreversibility or the position of the bump, shoulder, plateau, or glitch necessarily has the same shift exponent $2 / \phi$ !

One can treat the maxima ${ }^{10}$ in the singular part of $m$ (or $\chi$ ) in a similar way. Differentiating with respect to $T$ and equating the result to zero gives an equation of the form $g_{4}\left(h^{2}|t|^{-\phi}\right)=0$. For each solution $x_{i}$ of $g_{4}\left(x_{i}\right)=0$, the corresponding turning point follows $t \sim \pm h^{2 / \phi}$.

In fact, almost any line which originates from $t=0$ in a $h$-versus- $t$ plot and associated with the longitudinal magnetization has the same shift exponent $\phi$. However, as has been mentioned elsewhere, with features such as the maximum one must be careful to include the nonsingular terms. ${ }^{4}$

\section{CORRELATION LENGTHS AND FLUCTUATIONS}

Further new results and scaling laws are obtained by adding a few more physical ideas. If a spin glass does have a real phase transition there should exist a correlation length $\xi$ which diverges at $t=0$, i.e., $\xi \sim|t|^{-v}$ for $h=0$. In the presence of a field, one can think in terms of different longitudinal and transverse correlation lengths, with $\xi_{t} \sim\left|t^{\prime}\right|^{-v}$ and $\xi_{l} \sim|t|^{-v} g_{5}\left(h^{2}|t|^{-\phi}\right.$ ) (we shall derive this result below). In effect, at low field $h \ll|t|^{-\phi / 2}, \xi_{t}$ and $\xi_{l}$ behave similarly, but at high fields or low reduced temperature, $\xi_{l}$ saturates because the longitudinal transition is suppressed in a field.

At a given time the system might be pictured as composed of $n_{l}$ or $n_{t}$ more or less well-defined regions of size $\xi_{l}$ or $\xi_{t}$. Clearly $n_{l}\left(\xi_{l} / L\right)^{d}=n_{t}\left(\xi_{t} / L\right)^{d}=N$, the total number of spins, where $L$ is a typical spin-spin distance and $d$ the dimension of the system (possibly different from 3 because of fractal shaped clusters). In finite fields the response of the system depends upon the ratio of $\bar{m} h$ to the reduced temperature $\left(T / T_{G}\right)$, where $\bar{m}$ is the average magnetization associated with our above identified regions. Continuing this idea we might write

$$
m=n_{l} \bar{m} L\left(\bar{m} h T_{G}^{0} / T\right),
$$

where we have in mind that $L(x)$ is something such as the Langevin function. If $m$ is to have the scaling form of Eq. (17) we must require $\bar{m}=t^{-\phi / 2} g_{6}\left(h^{2}|t|^{-\phi}\right)$. Since $L(x)$ must be odd we have $L\left(\bar{m} h T_{G} / T\right)$ 
$=h|t|^{-\phi / 2} g_{7}\left(h^{2}|t|^{-\phi}\right)$, where both $g_{6}(x)$ and $g_{7}(x)$ are new scaling functions. The use of $n_{l} \sim \xi_{l}^{-d}$ and the substitution of it into (22) gives a scaling law $\phi+\beta=v d$ and

$$
\xi_{l}^{d}=t^{-v d} g_{5}\left(h^{2}|t|^{-\phi}\right)
$$

This confirms the expected behavior of the longitudinal correlation length. If we assume an asymptotic form $g_{5} \sim x^{b}$ as $x \rightarrow \infty$ and require $\xi_{l}$ to be independent of $t$ in the high-field limit, then we obtain $b=-(v d / \phi)$. Also for large fields we must require $m \sim h^{(2 / \delta+1)}$ and the function $L(x)$ should saturate as $x \rightarrow \infty$, giving $m \sim n_{l} \bar{m} \sim \xi_{l}^{-d} \bar{m}$. Then given $g_{6} \sim x^{a}$ as $x \rightarrow \infty$ we obtain $v d-\phi / 2+\phi(b-a)=0$ and $2 / \delta+1=-2(b-a)$. Eliminating $b-a$ yields the scaling law $v d=2 \beta+\gamma$, which by virtue of Rushbrooke's law $\alpha+2 \beta+\gamma=2$ (see Suzuki $\left.{ }^{5}\right)$ reduces to the well-known relation $v d=(2-\alpha)$. Finally, we solve for $a=-\frac{1}{2}$ and $b=-(1+1 / \delta)$ It is interesting that such precise results are obtained for $a$ and $b$, the high-field exponents for $\bar{m}$ and $\xi_{l}^{d}$, respectively.

This rather minimal physical picture also aids in the interpretation of the two crossover lines identified earlier. The high-temperature $t \sim h^{2 / \phi}$ crossover is associated with the experimental crossover from an analytic $h^{2}$ to nonanalytic $h^{2 / 8}$ dependence upon the field. ${ }^{1,3}$ The lowtemperature, $t \sim-h^{2 / \phi}$ line is more problematic. The irreversibility observed in a spin glass for $t<0$ and small fields is believed to be associated with strong replica symmetry breaking. Recently Parisi ${ }^{14}$ has brilliantly clarified the significance of this symmetry breaking. He describes it as a mathematical transcription of the existence of infinitely many pure thermodynamic states. A pure state corresponds to a single equilibrium state rather than a linear combination. He pictures configuration space to consist of many valleys, each the location of a pure state, separated by high free-energy barriers. Deep in the spinglass state a change in field results in a metastable state near the original pure equilibrium configuration. The decay through high barriers to the true equilibrium state takes a very long time, i.e., the system exhibits irreversible behavior. ${ }^{14}$ For large fields, in contrast, even for $t<0$, the effect of a changing field is merely to reorient the $n$ magnetizations $\bar{m}$ in a statistical way much as within standard Langevin theory. ${ }^{3}$ Some weak irreversibility is, however, necessarily associated with the reorientation of spins within a correlated region $\bar{m}$. We therefore identify the second low-temperature crossover as one from weak (or no experimental) to strong irreversibility, the generalization of the de Almeida-Thouless line. ${ }^{9}$

Also Parisi ${ }^{14}$ shows that the linear response of the system is related to the extremal value $q(1)$ of his function $q(x)$ which characterizes replica symmetry breaking. A full knowledge of $q(x)$ is only needed to describe the irreversible behavior. Our present non-mean-field approach, with its limited number of order parameters, should therefore be sufficient to describe linear response even in the presence of strong irreversibilities, without having to resort to multivalued scaling functions $\bar{\chi}(\bar{t})$.

\section{ACKNOWLEDGMENTS}

One of us (S.E.B.) acknowledges the partial support by National Science Foundation (Solid State Theory Program) Grant No. DMR-81-20827 and the kind hospitality of the IBM Thomas J. Watson Research Center, where his involvement in this work was initiated.
${ }^{1}$ A. P. Malozemoff, B. Barbara, and Y. Imry, J. Appl. Phys. 53, 2205 (1981).

${ }^{2}$ B. Barbara and A. P. Malozemoff, J. Less-Common Metals 9, 45 (1983).

${ }^{3}$ A. P. Malozemoff, S. E. Barnes, and B. Barbara, Phys. Rev. Lett. 51, 1704 (1983).

${ }^{4}$ B. Barbara, A. P. Malozemoff, and S. E. Barnes, J. Appl. Phys. 55, 1655 (1984).

${ }^{5}$ M. Suzuki, Prog. Theor. Phys. 58, 1151 (1977).

${ }^{6}$ M. Gabay and G. Toulouse, Phys. Rev. Lett. 47, 201 (1981).

${ }^{7}$ D. M. Cragg, D. Sherrington, and M. Gabay, Phys. Rev. Lett. 49, 158 (1982).

${ }^{8}$ K. H. Fischer, Phys. Rev. Lett. 34, 1438 (1975).
9J. R. L. de Almeida and D. J. Thouless, J. Phys. A 11, 983 (1978).

${ }^{10}$ H. Bouchiat, J. Phys. C 16, 445 (1983); Phys. Rev. B (to be published).

11J. Vanninenus, G. Toulouse, and G. Parisi, J. Phys. (Paris) 42, 565 (1981).

${ }^{12}$ W. E. Fogle, J. D. Boyer, R. A. Fisher, and N. E. Phillips, Phys. Rev. Lett. 50, 1815 (1983); I. A. Campbell, N. de Courtenay, and A. Fert, J. Phys. Lett. 45, L565 (1984).

${ }^{13}$ See, e.g., P. Pfeuty and G. Toulouse, Introduction to the Renormalization Group and to Critical Phenomena (Wiley, London, 1977).

${ }^{14}$ G. Parisi, Phys. Rev. Lett. 50, 1946 (1983). 